\title{
A Note on Translations
}

English translations of passages cited from original-language editions are my own. Other translations are from the cited published English editions. Full information on original and English editions is provided in the bibliography. 
